\title{
Les Maximes de La Rochefoucauld en tant que nouveau roman: entreprise phénoménologique?
}

Je ne me propose ni de trouver l'inspiration des nouveaux romanciers chez La Rochefoucauld ni de découvrir en lui le précurseur de la phénoménologie. Je cherche pourtant à établir des rapports entre les Maximes et I'entreprise phénoménologique du nouveau roman. Ces rapports ne se trouvent pas dans la modernité de La Rochefoucauld bien qu'il soit évident que son étude de 1 'homme peut s'appliquer aux hommes de cette époque. C'est plutôt dans la recherche de la réalité où l'ouvrage de La Rouchefoucauld et le nouveau roman se rapprochent 1 'un de $I^{\prime}$ autre.

Les nouveaux romanciers ont comme but le déchiffrement du réel, une recherche empruntée à la phénoménologie. Bien entendu, La Rochefoucauld ne savait rien d'une telle philosophie parce que ce n'était que pendant le vingtième siècle, grâce au philosophe allemand Husser1, que la phénoménologie est devenue célèbre. Je ne parle pas du phénoménisme de Kant qui croyait qu'il n'existe que des phénomènes et que $I^{\prime}$ homme serait donc incapable de rien savoir du réel. La phénoménologie va plus loin et, en disant qu'une réalité peut être atteinte, elle "se propose, par la description des choses ellesmêmes. - . de découvrir les structures transcendantes de la conscience. . . et les essences. Un phénoménologue croit donc que, par l'esprit, il révélera la constitution du monde.

II semble que La Rochefoucauld, à son tour, ait essayé de pénétrer au fond des choses pour y 
voir clair. C'est là où il rejoint les nouveaux romanciers. Cependant, la recherche de la réalité ne se limite pas du tout à La Rochefoucauld ni au nouveau roman. $\mathrm{Y}$ a-t-il un mouvement artistique ou littéraire qui ne veuille pas faire ressortir la réalité de sa propre façon? Je désire donc regarder de plus près les Maximes et quelques exemples du nouveau roman pour voir les points fondamentaux de cette recherche. C'est la manière de leur recherche plutôt que la recherche elle-même qui mérite d'être étudiée.

En bref, la phénoménologie est un moyen d'arriver à une réalité plus solide en "dégageant 1'immédiat de ce qui le dissimule."2 Eile admet qu'il existe un extérieur qui cache l'essentiel et qui fait augmenter de nombreux points de vue. La réalité peut donc devenir quelque chose de subjectif que chacun crée à son tour. La Rochefoucauld reconnaît ce problème quand il écrit: "Nos actions sont comme les bouts-rimés, que "3 chacun fait rapporter à ce qu'il lui plaît. Cette maxime résume en peu de mots la fausse réalité dont se plaignent les phénoménologues, une réalité qui change selon le désir des hommes. D'après La Rochefoucauld, une action, étant terminée, ne devrait pas se prêter à de diverses interprétations intéressées. Mais cela se fait. La Rochefoucauld se rend compte de la même réalité subjective dont parleront les nouveaux romanciers trois cents ans plus tard.

C'est la perception humaine qui agit sur ce qui devrait être invariable. Malheureusement, ce $n^{\prime}$ est qu'à travers la perception humaine que les choses sont vues. Les passions sont étroitement liées à cette perception et siont donc capables d'empêcher 1'homme de saisir ce qui est réel en s'attachant à sa manière de connaître le monde qui l'entoure. Quant aux passions, La Rochefoucauld constate: "Les passions ont une 
injustice et un propre intêrêt qui fait qu'il est dangereux de les suivre, et qu'on s'en doit défier, lors même qu'elles paraissent les plus raisonnables" (no. 9).

Les nouveaux romanciers veulent aussi peindre $1^{\prime}$ homme qui est dupe de ses passions et donc de ses perceptions. L'homme se trompe en dépit de son désir de raisonner son existence. Michel Butor présente un tel homme dans son roman, L'Emploi du temps. Jacques Revel se trouve obligé de travailler toute une année à un poste ennuyeux dans la ville imaginaire de Bleston. Pendant ce séjour il se croit héros dans deux différentes liaisons amoureuses; il se croit aussi responsable d'un attentat de meurtre. Pour Reve1, ces choses font la réalité même mais, au fur et à mesure que cette année s'écoule, il se rend compte qu'il n'est devenu $I^{\prime}$ amant de personne et que I'attentant de meurtre n'était probablement qu'un simple accident. Revel, tout au long du roman, s'érige en conducteur de sa vie et de ses propres expériences. I1 se met même à reconstruire par écrit tout ce qui lui est arrivé pour mieux supporter son existence dans cette ville qui le fascine et qui lui fait peur à la fois. Dans ce but, il essaie de s'insérer dans la vie quotidienne de quelques blestoniens. Cependant, il ne réussit pas à le faire parce qu'il passe trop de temps à êcrire ses aventures. Cette écriture est devenue, malgré lui, son seul but.

La Rochefoucauld décrit une situation pareille: "L'homme croit souvent se conduire lorsqu'il est conduit, et pendant que par son esprit il tend à un but, son coeur I'entraîne insensiblement à un autre" (no. 43). C'est la situation de Jacques Revel, un homme qui se laisse entraîner inconsciemment par son coeur jusqu'à ce qu'il soit trop tard. Son année à 
Bleston se termine et Revel manque les occasions de bonheur qui se présentent à lui à cause de cette manie imprévue de mettre par écrit sa vie. Revel, qui se croyait mâ̂tre de sa vie, trouve que "I'esprit est toujours la dupe du coeur" (no. 102). Ce problème de la perception humaine est cher à La Rochefoucauld qui en parle à travers ses Maximes.

Pourtant, ballotté entre ses perceptions trompeuses, comment est-ce que 1'homme voit les autres? La Rochefoucauld répond à cette question par sa première maxime:

Ce que nous prenons pour des vertus n'est souvent qu' un assemblage de diverses actions et de divers intérêts que la fortune ou notre industrie savent arranger, et ce n'est pas toujours par valeur et par chasteté que les hommes sont vaillants et que les femmes sont chastes. (no. 1)

Donc, il existe rarement des gestes désintéressés. Cela veut dire qu'il y a souvent une force cachée qui dirige chaque action humaine. On peut donc pénétrer les actions d'autrui, abordé par La Rochefoucauld, se trouve repris par le nouveau roman. Claude Simon, dans La Route des Flandres, présente un tel effort. Un homme qui s'appe1le Georges, pendant une seule nuit en 1946, est en train de se souvenir de ses expériences de la deuxième guerre mondiale. C'est la mort de son capitaine sept ans auparavant qui occupe ses pensées. Ce qui est intéressant, c'est que Georges passe cette nuit avec la veuve de son capitaine qui est devenue sa maîtresse. Le capitaine, par un geste de défi peut-être, s'était montré aux artilleurs allemands qui $1^{\prime}$ ont abattu aussitôt. Est-ce que c'était un suicide triomphant? Au 
début de la nuit Georges croit que son capitaine s'est tué en héros, un geste digne d'un vieux capitaine de cavalerie qui affront la guerre moderne. Cependant, après avoir réfléchi à ses propres motifs, en se demandant pourquoi il couche avec la veuve du capitaine, Georges finit par admettre qu'il n'en sait plus rien. Il est très possible que son capitaine ne soit pas mort en héros. Peut-être s'est-il laissé tuer exprès parce qu'il était victime d'une folie ou parce qu'il se doutait même à cette époque que sa femme lui était infidèle. Peut-être est-il mort à cause d'une bévue idiote.

La Rochefoucauld se rend compte d'un tel problème et il en admet la difficulté: "Pour bien savoir les choses, il en faut savoir le détail, et comme il est presque infini, nos connaissances sont toujours superficielles et imparfaites" (no. 106). Le monde, à cause de notre manque de détail, n'est presque jamais ce qu'il sembe être au premier abord. C'est là le deuxième lieu commun entre La Rochefoucauld et le nouveau roman.

Je viens d'étudier les deux pôles fondamentaux de la Rochefoucauld, I'homme qui se trompe et 1 'homme trompé, en les appliquant à quelques exemples du nouveau roman qui touchent aux mêmes problèmes. Ces deux pôles sont la raison d'être de leur recherche phénéménologique. Les Maximes et le nouveau roman se rejoignent donc par leur introduction systématique du doute en ce qui concerne la perception humaine. Même au début des Maximes, La Rochefoucauld souligne fortement ce doute quand il met en exergue le proverbe qui révèle au lecteur la vraie motivation derrière un geste ou une conduite qu' on croit sincères: "Nos vertus ne sont le plus souvent que des vices déguisés." 


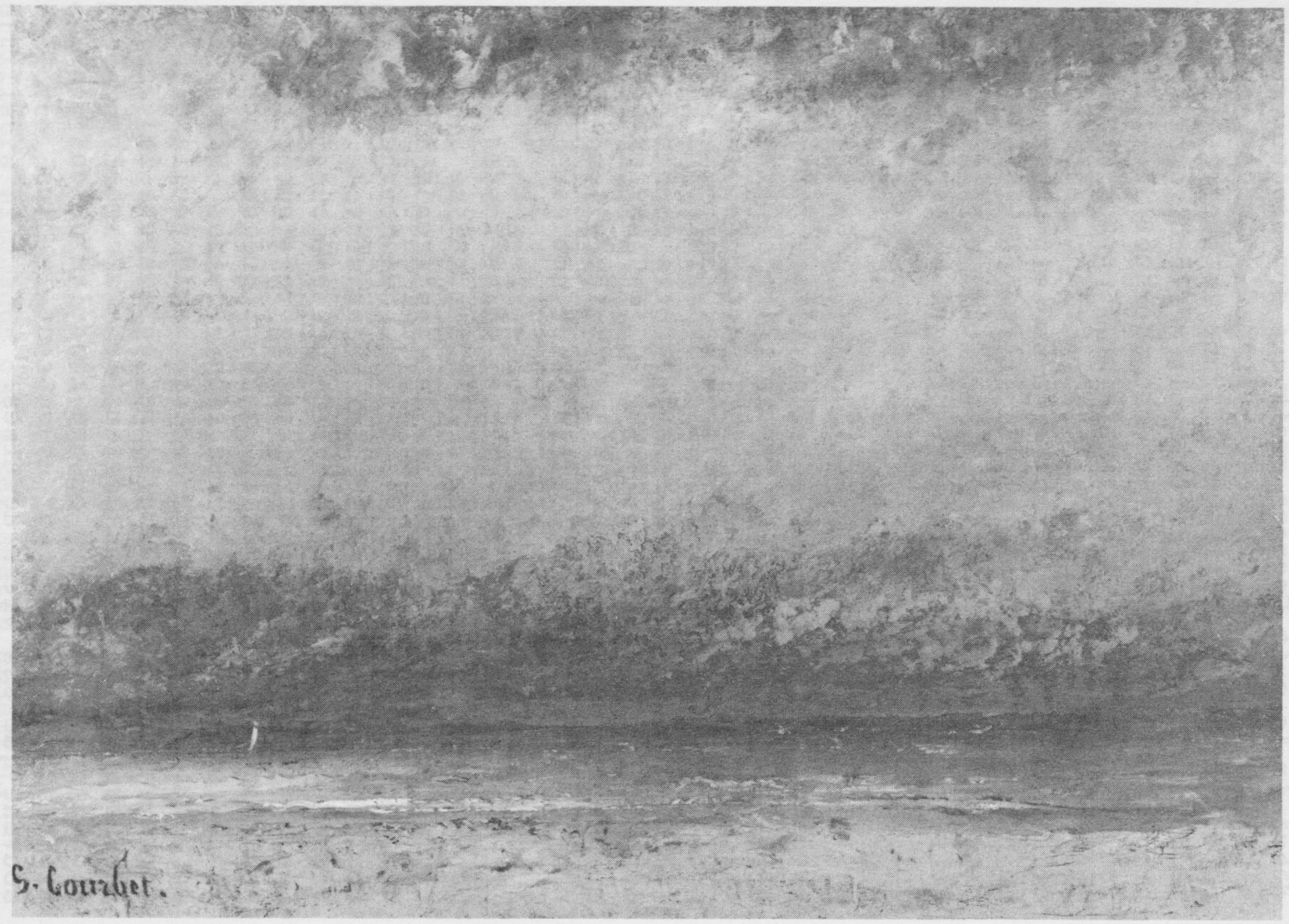


Pourtant, les procédés de La Rochefoucauld ressemblent-ils tout à fait à ceux des nouveaux romanciers? Les Maximes ne comprennent pas de roman qui doit être lu du début jusqu'à la fin pour se faire comprendre. Les nouveaux romanciers s'appuient cependant beaucoup sur la forme classique du roman. C'est-à-dire, le roman qui se lit d'une manière logique de la première page jusqu'à la dernière. Les nouveaux romans ne sont pas destinés à être feuilletés au hasard. A la différence d'un nouveau roman, les Maximes peuvent se prêter à une lecture au hasard sans vraiment perdre de signification. Mais ce n'est pas à dire que La Rochefoucauld veuille que son ouvrage se lise de cette manière. Malgré ce qu'il dit dans son avis au lecteur, il est très probable que La Rochefoucauld a beaucoup travaillé sur 1 'ordre des Maximes.

Donc, il se sert peut-être de quelques procédés stylistiques pour unifier cet ouvrage tandis qu'il attire le lecteur. Peut-on dire que La Rochefoucauld se répète à dessein? Il faut regarder quelques-unes de ses maximes qui traitent le même sujet. Après avoir abordé les vertus tout au début des Maximes, La Rochefoucauld les regarde à plusieurs reprises à travers son ouvrage:

Pendant que la paresse et la timidité nous retiennent dans notre devoir, notre vertu en a souvent tout l'honneur. (no. 169)

Le nom de la vertu sert à l'intérêt aussi utilement que les vices. (no. 187)

La vertu n'irait pas si loin si la vanité ne lui tenait compagnie. (no. 200) 
L'intérêt met en oeuvre toutes sortes de vertus et de vices. (no. 253)

La Rochefoucauld change souvent de sujet pour garder 1'attention du lecteur mais, en fin de compte, il se répète. Il ne faut que regarder un index thématique des Maximes pour en voir la preuve. Cette répétition sert sans doute à mieux atteindre ce lecteur qui lit et relit de $I^{\prime}$ amour, de l'esprit, de I'intérêt, des passions, de la vertu, etc. C'est cependant plus que les termes exacts qu'il remploie; il y a aussi une répétition des idées. Par exemple, sans mentionner la vertu, La Rochefoucauld y fait parfois allusion: "I1 est difficile de juger si un procédé net, sincère et honnête est un effet de probité ou d'habiletè" (no. 170) C'est grâce à cette répétition que Ia Rochefoucauld se fait mieux comprendre. Il dessine, en effet, un cercle fermé où se trouve 1'homme.

Ce procédé, celui de la répétition et de l'accumulation, s'emploie couramment dans le nouveau roman. Par la répétition, les nouveaux romanciers désirent peindre l'homme en train de se rendre compte que la vie se réduit à quelques points fondamentaux. En voici quelques exemples que Michel Butor nous donne:

Sur le tapis de fer chauffant, vous voyez un pépin de pomme sauter d'un losange à un autre.

Sur le tapis de fer chauffant il y a deux pépiris immobiles tout à côté de votre pied gauche. (p. 132)

Sur le tapis de fer chauffant. . . les deux pépins de pomme sont écrasés sur une rainure. (p. 134) 
Ensuite, il est de toute évidence que les Maximes visent 1'espèce humaine. Il est rare que La Rochefoucauld décrive un objet ou quelque chose de la vie quotidienne s'il ne les emploie pas pour mieux s'exprimer. Par exemple: 'L' absence diminue les médiocres passions et augmente les grandes, çomme le vent éteint les bougies et allume le feu" (no. 276).' Et du même genre:

"La flatterie est une fausse monnaie qui n' ai de cours que par notre vanité" (no. 158). De tels exemples manquent à cet ouvrage de La Rochefoucauld parce que, se bornant à l'homme, il ne passe pas beaucoup de temps à parler des bougies ni de la fausse monnaie.

$D^{\prime}$ autre part, il semble qu'il y ait une présence obsédante des objets dans le nouveau roman. Les nouveaux romanciers $n$ 'hésitent pas à décrire en profondeur les objets les plus insignifiants qui ne renvoient à rien:

Un quartier de tomate en vérité sans défaut, découé à la machine dans un fruit d'une symétrie parfaite.

La chair périphérique, compacte et homogène, d'un beau rouge de chimie, est régulièrement épaisse entre une bande de peau luisante et la loge où sont rangés les pépins, jaunes, bien calibrés, maintenus en place par ung mince couche de gelée verdâtre. . .

Est-ce que cela veut dire que La Rochefoucauld essaie d'étudier 1'homme tandis que les nouveaux romanciers ne veulent décrire que le monde qui $I^{\prime}$ entoure? Pas du tout. Les objets dans un nouveau roman ne sont vus que par l'homme et c'est donc à travers $I^{\prime}$ homme que le monde se forme: 
L'homme y est présent à chaque page, à chaque ligne, à chaque mot. Même si $1^{\prime}$ on trouve beaucoup d'objets, et décrits avec minutie, il y a toujours et d'abord le regard qui les voit, la pensée qui les revoit, la passion qui les déforme. Les objets de nos romans $n$ 'ont jamais de pré- 6 sence en dehors des'perceptions humaines.

Il est de plus en plus évident que La Rochefoucauld et les nouveaux romanciers se ressemblent en ce qui concerne leur attitude envers la perception humaine. Cette perception, à cause du fait qu'elle est humaine, obscurcit le monde. En conséquence, I'homme se trompe et se laisse tromper. Puis, en dépit de leurs structures différentes, les Maximes et le nouveau roman emploient des procédés pareils, la répétition et $1^{\prime}$ accumulation, pour renforcer les images qu'ils présentent au lecteur. Enfin, La Rochefoucauld et les nouveaux romanciers ne se consacrent qu'à $I^{\prime}$ homme parce que c'est grâce à l'homme que le monde a sa valeur.

Cependant, à part ce déchiffrement du réel, peut-on dire que c'est vraiment une entreprise phénoménologique? Il ne faut pas regarder les rapports entre la phénoménologie et le nouveau roman parce que cela se voit non seulement par les connaissance personnelles que certains nouveaux romanciers ont de la phénoménologie, mais par I'intérêt qu'ont pris aux nouveaux romans certains phénoménologues officiels. 7 Quant à La Rochefoucauld, c'est ce qu'il reste à voir. II vaut mieux étudier en ce moment une méthode aux Maximes et au nouveau roman pour faire ressortir les ressemblances. Dans ce but je me propose de regarder un manuscrit de Gaston Berger, un phénoménologue français qui était actif juste avant et pendant les années 
cinquante, la période de la naissance du nouveau roman. Dans ce manuscrit se trouve la méthode phénoménologique qu'il a développée après tant d'études.

\$Etudier des cas \&oncrets. Les voir simplement, tels qu'ils sont.

Chaque maxime de La Rochefoucauld aborde un certain cas précis, chaque maxime ayant son propre sujet qui est soit dit soit suggéré. Par exemple: "Les hommes ne vivraient pas longtemps en société, s'ils n'étaient les dupes les uns des autres" (no. 87). En ce qui concerne cette maxime, le sujet se trouve écrit: la société. La Rochefoucauld se borne à commenter brièvement cela en disant que la société des hommes se base sur la duperie mutuelle.

Ensuite: "Nul ne mérite d'être loué de bonté, s'il n'a pas la force d'être méchant; toute autre bonté n'est le plus souvent qu'une paresse ou une impuissance de la volonté" (no. 237). C'est un autre cas concret, le sujet étant la faiblesse dont parle La Rochefoucauld sans le dire directement. II explique en peu de mots que 1'homme a souvent 1'air bon à cause de sa faiblesse. Une telle étude, la première démarche de la recherche phénoménologique, continue tout au long de 1 'ouvrage.

Quant au nouveau roman, il ne faut que regarder 1 'intrigue de quelques exemples de ce mouvement. Le personnage principal de Quelqu'un de Robert Pinget passe toute sa journée à chercher un bout de papier qu'il vient de perdre. En fouillant la maison et le jardin, il essaie de reconstruire ce qu'il a fait juste avant et après la découverte de sa perte. Dans La Marquise sortit à cinq heures, Claude Mauriac présente les pensées d'un homme qui reste debout toute une 
heure devant sa fenêtre. Enfin, dans La Modification de Miche1 Butor, Léon Delmont voyage de Paris à Rome par le train tandis $\mathrm{qu}^{\prime} i 1$ revoit son passé et pense à son avenir. I1 ne quitte guère son compartiment. Evidemment, en évitant les péripéties, les nouveaux romanciers savent aussi se borner à des cas simples.

§Pratiquer la réduction historique. Ecarter les théories, les systèmes, les idées préconçues. Voir les choses elles-mêmes.

Quand quelqu'un fait du bien, celui-ci en fait soit à dessein soit malgré lui. Cependant, en faisant du bien, on est censé être généreux. C'est $1^{\prime}$ opinion que vise La Rochefoucauld quand il écrit: "La clémence des princes n'est souvent qu'une politique pour gagner 1'affection des peuples" (no. 15). I1 dit, en effet, qu'il faut se méfier des pensées provenant de la coutume et des idées traditionnelles parce qu'elles nous empêchent de voir vraiment le monde. Le mot même de "clémence" déguise trop d'actions intéressées.

Un autre exemple du danger des idées préconçues serait ceci: "La constance des sages n'est que $1^{\prime}$ art de renfermer leur agitation dans le coeur" (no. 20). La Rochefoucauld reconnaît que I'homme se laisse aveugler par les termes qu'il donne aux apparences parce que ces termes, renforcés par un long emploi, cachent ce qui est l'essentiel d'une action.

Les nouveaux romanciers se rendent compte du même problème et, en conséquence, ils veulent repenser les termes dont ils ont hérité:

Mais nous sommes tellement habitués à entendre parler de personnage, d'atmosphère, de forme et de contenu. . . qu'il nous faut un effort pour nous dégager de 
cette toile d'araignée et pour comprendre qu'elle représente une idée sur le roman (idée toute faite, que chacun admet sans discussion, donc idée morte). .

§L'ingénuité du regard est une conquête. Voir "neuf." (B)

Existe-t-i1 des maximes qui ne voient pas neuf? La Rochefoucauld frappe le lecteur par ses observations qui ne répondent guère à ce $q u^{\prime}$ on accepte pour vraies. Son ingénuité du regard est souvent une continuation de sa réduction historique. Il détruit donc une idée fixe quand il dit: "Si on croit aimer sa mâtresse pour I'amour d'elle, on est bien trompé" (no. 374). Qui penserait $q u^{\prime}$ on aime une maîtresse pour d'autres raisons avant de lire un telle maxime?

Ensuite: "Le refus des louanges est un désir d'être loué deux fois" (no. 149). Tout le monde semble refuser des louanges pour ne pas apparaître immodeste mais, selon La Rochefoucauld, on se trompe. Quiconque lit cette maxime se rendra compte de sa vraie motivation la prochaine fois qu'il se trouve en train de refuser une louange. C'est encore l'ingénuité de La Rochefoucauld qui contraint son lecteur à réfléchir. Ce n'est pas à dire que La Rochefoucauld ait toujours raison; il faut remarquer cue l.a maxime ne propose pas de solution. Pourtant, grâce à son ingénuitê du regard, la réalité d'une situation commence à se dévoiler.

Cette idée de voir neuf est une des bases du nouveau roman pour éviter le regard traditionnel du roman qui $1^{\prime} a$ précédé. Il $n^{\prime} \mathrm{v}$ a plus de narrateurs omniscients qui racontent tout ce qu'il faut savoir. Ise plus souvent, le lecteur d'un nouveau roman ne voit qu'à travers les yeux 
du personnage principal parce que le narrateur traditionnel 'he voit et ne reproduit que les grandes lignes immobiles;. . . il reconnaît parmi elles celles qui sont déjà expliorées et les désigne par leurs noms connus."I0 A la manière de La Rochefoucauld, les nouveaux romanciers se servent de cette "nouveauté" du regard pour aider le lecteur à s'approcher de la réalité.

\$Eliminer 1'accidental. Chercher I'essence. Eliminer le superficiel, le fortuit, le pittoresque.

Les différentes éditions des Maximes témoignent du fait que La Rochefoucauld a passé beaucoup de temps à refaire son ouvrage pour atteindre la meilleure expression avec un minimum de mots. Jean-Pol Caput, dans son édition des Maximes, démontre le développement du numéro 186:

Avant 1665: On hait souvent les vices, mais on méprise toujours le manque de vertu.

Edition de 1665: On peut haĩr et mépriser les vices, sans haĩr ni mépriser les vicieux, mais on a toujours du mépris pour ceux qui manquent de vertu.

Edition de 1678: On ne méprise pas tous ceux qui ont des vices, mais on méprise tous ceux qui n'ont aucune vertu.

Personne n'accuserait La Rochefoucauld d'être pittoresque et, comme nous $I^{\prime}$ avons déjà remarqué, il n'emploie des exemples mondains que pour mieux s'exprimer. Donc, quand il élimine tout ce dont le lecteur n'a pas besoin pour comprendre une maxime, il s'avance vers I'essence 
de son sujet. Quand La Rochefoucauld refuse 1'accidental et le superflu, il finit par maîtriser son art.

Au premier abord, le style des nouveaux romanciers n'est pas aussi lapidaire que celui de La Rochefoucauld; il semble que leurs descriptions continuent à jamais. Cependant, ils se bornent de la même façon que lui parce qu'ils refusent à leur tour tout ce qui n'est pas nécessaire au roman. Les nouveaux romanciers décrivent beaucoup mais leurs descriptions sont un moyen de réduction pour saisir la réalité, une entreprise phénoménologique parce que la phénoménologie est surtout destinée à rendre compte "de 1'espace, du temps, du monde vécus"12 à travers une description qui est la plus pure.

§Procédé: variation des exemples. Voir, à travers la variation, la permanence de la loi profonde.

(B)

La variation chez La. Rochefoucauld n'est pas la même chose que la répétition. Variation veut dire le même sujet traité de plusieurs façons et non pas le même sujet répété plusieurs fois. A vrai dire, à partir de son exergue, La Rochefoucauld ne ramasse que des exemples d'un monde qui n'est pas le plus souvent ce qu'il paraît être. Ce qui rend son ouvrage important, c'est sa capacité de regarder infiniment la même chose à travers tous les sujets qu'il traite. En fin de compte, il revient toujours à ce premier problème qui se présente dans son exergue et qui résume tout La Rochefoucauld. Quant au nouveau roman:

- . Ia technique du redoublement s'avère donc comparable, sinon analogue, au processus phénoménologique de la 
variation libre qui consiste à représenter un certain objet. . . sous tous ces aspects possibles, mais seulement sous des aspects possibles car il y a des limites que ce processus se peut dépasser.

La Rochefoucauld se donne des limites pareilles quand il dit: "Le plus grand défaut de la pénétration n'est pas de n'aller point jusqu'au but, c'est de le passer" (no. 377).

Aussi existe-t-il des rapports entre La Rochefoucauld et les nouveaux romanciers. Bien entendu, ce sont des coincidences mais ce sont des rapports importants quand même. Tous les deux désirent écarter d'une manière semblable le voile qui se trouve entre $1^{\prime}$ homme et la réalité en démontrant la nature trompeuse des perceptions humaines. Pourtant, ils se rendent compte que ce $n^{\prime}$ est $q u^{\prime}$ à travers la perception humaine $q u^{\prime} i l s$ connalssent et font connaitre le monde. C'est donc une étude qui ne vise que I'homme.

En dernière analyse, il est évident que La Rochefoucauld et les nouveaux romanciers ne se croient pas capables d'arriver à une réalité absolue. Quant à la description phénoménologique du nouveau roman, sa caractéristique principale "est de ne plus chercher à transmettre un savoir positif sur les choses, mais à montrer comment 1 'homme perçoit et connaĩt le monde. "14 Voici le dernier et le plus important lieu où La Rochefoucauld rencontre les nouveaux romanciers et donc leur entreprise phénoménologique. La Rochefoucauld observe et décrit. Mais il n'essaie pas d'indiquer au lecteur ce $q u$ 'il doit faire; il ne lui indique que ce qu'il fait. C'est par ce portrait de la nature humaine et non pas par des 
solutions toutes faites que le lecteur finit par se voir et se comprendre. Ce n'est pas à dire que La Rochefoucauld n'essaie jamais d'interpréter ce $q u^{\prime} i l$ observe. Cependant, à cause de sa forme cațégorique et souvent déroutante, aucune maxime "ne peut prétendre. . . apporter une vérité définitive sur tel ou tel aspect de 1 'homme." 15 Bien qu'il ait devancé par trois cents ans la phénoménologie et le nouveau roman, La Rochefoucauld a entrepris ce qui s'appellerait une recherche phénoménologique.

\author{
WILLIAM HELLING \\ UNIVERSITY OF KANSAS
}


NOTES

1 Phénoménologie, Le Petit Robert, éd. 1970, p. 1292 .

${ }^{2}$ raston Berger, Phénoménologie du temps et prospective (Paris: Presses Universitaires de France, 1964), p. 51.

${ }^{3}$ François de La Rochefoucauld, Réflexions ou maximes morales, éd. Pierre Kuentz (Paris: Bordas, 1966), no. 382. Toutes les citations des Maximes renvoient à cette édition.

4 Michel Butor, La Modification (Paris: Les Editions de Minuit, $\overline{1965), p .124 . ~ L e s ~ d e u x ~}$ citations suivantes renvoient aussi à cette édition.

${ }^{5}$ Alain Robbe-Grillet, Les Gommes (Paris: Les Editions de Minuit, 1953), p. 161.

${ }^{6}$ Alain Robbe-Grillet, Pour un Nouveau Roman (Paris: Les Editions de Minuit, 1963), p. 116.

${ }^{7}$ Pierre A.-G. Astier, La Crise du roman français et le nouveau réalisme (Paris: Nouvelles Editions $\overline{\text { Debresse, 1969), p. } 295 .}$

8 Berger, p. XI. Toutes les démarches de cette méthode à ce manuscrit et ci-après seront indiquêes par la lettre $B$. 
${ }^{9}$ Robbe-Grillet, Pour un Nouveau Roman, p. 25.

10 Nathalie Sarraute, L'Ere du soupçon (Paris: Librairie Gallimard, 1956), p. 115.

${ }^{11}$ Jean-Paul Caput, éd. dans François de la Rochefoucauld, Maximes et réflexions diverses (Paris: Librairie Larousse; Nouveaux Classiques Larousse, 1975), p. 15.

${ }^{12}$ Maurice Merleau-Ponty, Phénoménologie de 1a perception (Paris: Librairie Gallimard, 1945), p. 1 .

${ }^{13}$ Astier, p. 303

14 Jean Thoraval, Nicole Bothere1, et Francine Dugost, Les Nouveaux Romanciers (Paris: Bordas, 1976), p. 104 .

${ }^{15}$ Caput, p. 15 . 